\title{
Use of Lean Robotic Communication to Improve Social Response of Children with Autism
}

\author{
Full Paper \\ Amir Talaei-Khoei \\ University of Nevada, Reno, USA \\ University of Technology, Sydney, Australia \\ atalaeikhoei@unr.edu \\ Mala Kaul \\ University of Nevada, Reno, USA \\ mkaul@unr.edu \\ Lundy Lewis \\ Southern New Hampshire University, \\ USA \\ l.lewis@snhu.edu \\ Jay Daniel \\ University of Technology, Sydney, \\ Australia \\ Jay.Daniel@uts.edu.au \\ Rajeev Sharma \\ University of Waikato, New Zealand \\ rsharma@waikato.ac.nz
}

\begin{abstract}
In light of inconclusive results reported in the literature on the benefits of using robots to foster social skills in autistic children, this paper assesses the use of robots with no facial expressions to create basic structured communication with autistic children. We address the complexity of communication when autistic children cannot understand the unintentional facial expressions of human instructors in training sessions. The paper reports 19 training sessions of a mild autistic child interacting with a humanoid robot with approximate duration of 20 minutes each. It was observed that during these 19 sessions, the child improved his responses to the directives given by the robot. The paper discusses the results in terms of the implications for professionals in the field. Further, the study serves as a proof of concept for future contributions to media richness theory.
\end{abstract}

\section{Keywords}

Lean Communication, Robots, Autism.

\section{Introduction}

Autism Spectrum Disorders (ASD) are neurodevelopmental conditions characterized by persistent and significant impairment in the social-communication domain (Association of Autistic Patients, 2015). The most recent reports released by the Centers for Disease Control and Prevention (CDC) estimate that as many as 1 in every 68 children are affected by ASD. ASD clinical features typically arise in the early developmental period of children. One of the debilitating conditions associated with ASD is the lack of social communications (Kroeger et al., 2007). As a result, these children are often targets of bullying and social rejection, which further increase their social isolation and takes a tremendous toll on their emotional and cognitive well-being (Cappadocia et al., 2012). While specific causes for ASD have yet to be found, very early diagnosis and intervention are the main approaches to managing the condition (Pennisi et al., 2015). There has been a body of literature on the necessity of social skills training for children with ASD (Bellini and Peters, 2008; Otero et al., 2015). Rapid progress in technology, especially in the area of robotics, offers promising possibilities for innovation in ASD interventions.

A key disability associated with ASD is the difficulty to engage in social communication and interaction. That has been attributed to a difficulty in interpreting facial expressions, which could contribute to their failure to understand verbal messages and engage in social communication and interaction (Hobson, 
1986; Russo-Ponsaran et al., 2015). The facial expressions that instructors unintentionally show make communication too complex for the child, with the child eventually losing interest in and abandoning the interaction (Otero et al., 2015). Consequently, those children typically face difficulties in engaging in social communication and interaction.

Prior literature has argued that the use of robot can be a powerful support for engaging with children with ASD (Ricks \& Colton, 2010), and also developing their skills for social communication and interaction (Kim et al., 2012). A key argument underpinning that approach is that robotic communication can be designed to minimize additional cues, such as facial expressions and tone of voice, that characterize human communication. Consequently, children with ASD are less likely to be overwhelmed by the information over-load that human communication often imposes on them and are more likely to respond to and interact with robotic communication.

This paper describes an exploratory research designed to understand the effect of lean robotic communication on the interactive responses of children with ASD, for developing a training program to improve their communication and interactional skills, and tools for diagnosing the extent of autism and measuring improvements in their responses. We describe the design and results of a study conducted over a period of five months that assessed the communication between a subject with ASD and a robot. The experiment measured the performance and progress of the subject over the five months. The findings support the use of lean communication methods to engage more effectively with children with ASD.

The authors appreciate the limitations involved with having only one subject; however, this research was conducted in a rigorous manner and the promising results from this pilot study contribute to the ASD literature by providing an avenue for further research in the application and use of lean communicating humanoid robots for improving social communication in children with ASD. Further, the study leads us to question certain assumptions of media richness theory, which finds that task characteristics and communication media determine correct media fit (Suh, 1999). Our results extend media richness theory and propose that the recipient's social characteristics also figure into the determination of media fit. This research will be followed by comparative studies of diverse participant groups in future experiments.

The rest of this paper is organized as follows: The following section reviews the literature and presents the background for this study. Next, we present the methods section followed by the results. Finally, we wrap up with a discussion of our findings, the limitations of the pilot study, and describe the next steps in our research.

\section{Background}

In this section we first examine the literature pertaining to robotic interactions for ASD therapy. Next we find support for lean communication in preventing sensory overload. This leads us to propose the use of lean communication based robotic interaction, for improving social communication in children with ASD.

\section{Use of robots for ASD therapy}

Much of the literature discussing robots and children with ASD, has focused on comparing the communication between robot instructors and human instructors. While some studies (Yee et al., 2012; Yin and Tung, 2013; Zheng et al., 2013) showed mixed results in the advantages of robots in training sessions with ASD children, recent studies have demonstrated that a robot can provide better stimulus than a human instructor in improving social behaviors in children with ASD (Anzalone et al., 2014; Dautenhahn, 2007; Scassellati et al., 2012; Wainer et al., 2014). A critical review of the literature on robotic interactions with ASD children, found many potential advantages to using interactive robots in clinical settings, such as, the intrinsic appeal of technology, robots' ability to produce simple social behaviors repetitively, and that the robot programming could be individually adapted for personalized treatment (Diehl et al 2012). While a large number of studies pertaining to robotic interactions with ASD children focus on the development of robotic technology, there is less focus on the clinical application of robotic technology. Further, the role of robots as a supporting tool for instructors to develop a standardized social context in the training sessions has been largely ignored (Pennisi et al., 2015). This 
research aims to address this gap in the literature, by examining the application and impact of robots on social interactions in the case of children with ASD.

\section{Non-humanoid robots versus humanoid robots}

In a more specific measure, Puyon and Giannopulu (2013) believe that children with autism do not show any social behavior or reactions towards the robots. However, the robots that were used in their study were non-humanoid, and as such it is difficult to generalize these findings. Therefore, an interesting line of research is to test whether similar results would be observed by using a humanoid robot. Thus, the aim of our study is to provide a social context using a humanoid robot in training sessions and to measure the progress of the subjects. Cabibihan et al. (2013) defines the technical features of robots that have been used for ASD intervention along three dimensions: (1) design features that need to be optimized to meet the needs of individuals with ASD (physical appearance, functionality, level of autonomy, etc.), (2) the different roles that robots can play in ASD interventions, and (3) the activities that should be carried out during the therapy. This work directs us in the choice of the $\mathrm{NAO}^{1}$ humanoid robot as an interactive partner in the training sessions. Although some studies have used animal-like robots (Diehl et al 2012; Scassellati et al., 2012), humanoid robots have been found to relate better to autistic children in social contexts Pennisi et al. (2015). Therefore, inspired by this work, we include three types of activities attention, joint-attention and turn-taking in our study of the social interaction of a humanoid robot with ASD children.

\section{Facial expression can confuse children with autistic}

Although the use of humanoid robots seems more promising than non-humanoid robots, children with ASD, are generally known to have challenges in recognizing complex facial and gestural expressions and difficulties associating facial emotional expressions during a conversation. (Robins et al. 2009). Robins et al. $(2005,2006)$ found that robots that interact with children with ASD should avoid the details and complexity of a human while still holding to the humanoid form. Scassellati (2007) emphasizes that structured interactions without complexity of humans' social behaviors (e.g. facial expressions) can create basic communication that is easier for children with ASD. Further, the literature suggests that the simplified social cues presented by robots could potentially result in less overstimulation of the children, and that robots could offer more predictable and better triggers for social behavior than the nuanced and subtle social prompts from a human partner (Scassellati et al. 2012). Therefore, the objective of this work is to assess the use of robots, with no facial expression, as a supporting tool for instructors in social skills training sessions. Consequently, we plan the use of (humanoid) robots that look and behave like a human instructor, and select robots that do not show any facial expression.

\section{Lean Communication}

Daft and Lengel (1986) in the early proposal of Media Richness Theory suggests that communication media have different capacities for resolving ambiguity, negotiating interpretations, and facilitating understanding. Suh (1999) proposes the Media Fitness construct that is influenced by characteristics of the task that is being communicated as well as the medium that is used for the communication. Better performance and more satisfaction is achieved when the medium is a better fit for the task at hand. Bubaš (2001) states that facial expressions and emotional signals create richer media, which can reduce misunderstandings and improve the quality of communication. Here, the main assumption of media richness theory is that the more cues the media can support, the richer the media is. In a number of instances, media richness theory has been questioned. However, the different sensory perceptions in children with ASD (Bogdashina 2016), indicates that in certain cases lean communication would be more appropriate. Consequently, the assumptions of media richness theory may need to be revisited.

Against this backdrop, the objective of this study is to examine the application and impact of humanoid robots, without facial expressions on social interactions in the case of children with ASD. Specifically, this paper addresses the following research question:

\footnotetext{
${ }^{1}$ NAO Specification. www.doc.aldebaran.com/2-1/family/nao_h25/index_h25.html. Retrieved 2/28/16
} 
Does the use of lean robotic communication improve the social response of children with ASD?

To address this question, we conduct a pilot study, over a period of five months, using a humanoid robot without facial expressions for social skill training of a child with ASD. The main objective is that the robot does not show facial or gesture expressions and maintains the communication at a basic level. Therefore, a child should be able to follow training until the end of a session, thus enhancing social skills that may transfer to real human interaction.

\section{Methods}

\section{Designing the Pilot}

The pilot study included nineteen training sessions, of approximately 20 minutes with the presence of a kid with autism, a humanoid robot, the operator of the robot and an instructor. The sessions took place in a common playroom of an elementary school. The instructor was the subject's Speech and Language Pathologist (SLP).

Subject of under pilot study: The purpose of the pilot study was to demonstrate the use of robots' lean communication for improving social response of children on the spectrum of ASD. As such to server the purpose, we had only one subject with mild ASD selected by the SLP. The subject is a 7-year old male in the 2nd grade. His SLP describes him as showing deficits in maintaining attention, joint attention, and taking initiative. He participates in special and general education classes, and receives direct instruction and gets support from paraprofessionals. He participates in individual and group speech therapy sessions. He plays games, watches TV shows, and scripts them both physically and mentally, showing a strong cognitive inner life. He reads at 1st to 2nd grade level but comprehension is at kindergarten level.

The authors acknowledge the limitation associated with pilot study of only one subject in ASD spectrum, particularly when analyzing the growth and improvement. However, the present paper serves to demonstrate the relevance of the idea. We plan to re-run the study on a larger number of participants with diverse social abilities, thereby improving the reliability of the results.

Robot: We used the NAO humanoid robot as an interactive partner in the social training sessions. NAO is a two-foot tall ten-pound humanoid robot with facilities for speech recognition, object recognition, mobility, and gesturing.

Settings: For each session, the robot was placed on a table. The subject and the SLP sat in chairs facing the robot. The robot was tele-operated by a robot operator sitting to the side of the robot. Figure 1 shows the setting for each training session; See Figure 1.

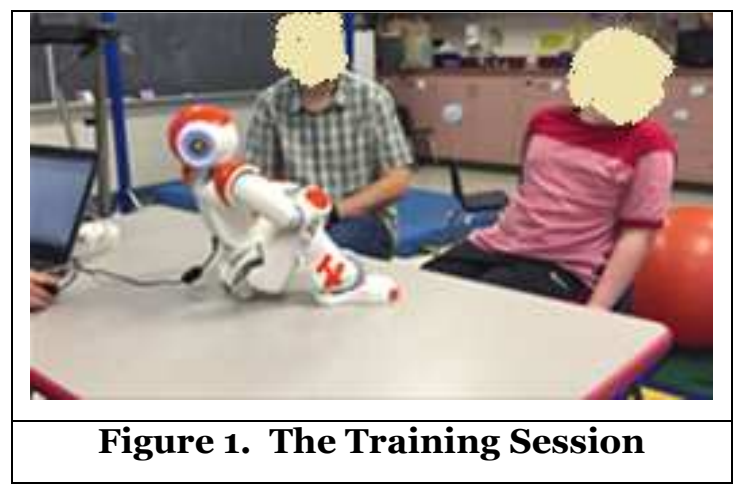

Sessions: During sessions, the robot provided directives to the subject to which the subject is expected to respond; however, the instructor may prompt the subject verbally or via gesturing if a response is not forthcoming. As a simple example in joint attention, the robot might say "Everybody look at the chalkboard," following which the subject is expected to turn his head toward the chalkboard. If he does not after a few seconds, the instructor may encourage the subject verbally, through touch, and/or gesturing. 
Lewis et al. (2016) states the importance of three types of directives and activities that need to be taken into account during the social training sessions of autistic subjects; namely attention, joint-attention and turn-taking. The sessions in our pilot study included these three types of activities. The attention directives were such that the robot asked the subject to perform a particular task. An example of a directive for attention is "[name], what does the pig say?" and "[name], tickle my belly" and the like. The joint attention directives were such that the subject pays attention to what others (the instructor and the robot) are attending to. An example of joint attention is "Everybody look at the chalkboard" and "[name], what am I looking at?" where the robot looks at the window. The turn taking activities are designed for social comprehension of the subject. An example of turn-taking is simply "[name], now it is your turn" during the "What does the X say?" game and the "Everybody look at X" game. Turn-taking is difficult for ASD children.

\section{Data Collection and Analysis}

All the sessions were filmed and analyzed by the authors. In order to analyze the performance of the subject, the following quantitative measures were extracted for each social training session.

- The number of total directives: The total number of directives the robot issued to the subject.

- The number of responses without prompts: The number of directives to which the subject responded correctly with no prompts from the instructor.

- The number of responses with prompts: The number of directive to which the subject responded correctly but only with prompts from the instructor, e.g. encouragement via verbalizing and gesturing.

- The number of no response: The number of directives to which the subject did not respond after a reasonable amount of time.

Then, a non-parametric trend analysis using Sen Slope test (Gilbert, 1987) was performed to demonstrate the growth or drop for each of these variables. The growth in the number of responses without prompting would indicate improvement in performance. However, this variable has negative inter-correlation with the sum of the other two variables.

\section{Results}

Table 1 presents the results of the pilot study. The table depicts the number of directives to which the subject responded without needing the instructor's encouragement, the number of directives to which the subject responded but only with the instructor's encouragement, and the number of directives to which the subject did not respond even with the instructor's encouragement. For all three measures, both the actual number as well as the percentage of total number of directives are reported. We note that sessions $1-8$ involved attention and joint attention activities where one can see visually a trend of improvement. Starting with the session 9, at the suggestion of the SLP we gradually introduced turn-taking directives. Although the total number of directives begin to drop at the session 9, one can see visually that the percentage of responses without instructor prompting does not drop and begins to increase gradually.

\begin{tabular}{|c|c|c|c|c|c|c|c|c|}
\hline \multicolumn{9}{|c|}{ Table 1. Results of the Pilot Study } \\
\hline & \multirow[t]{2}{*}{ Sessions } & \multirow[t]{2}{*}{$\begin{array}{c}\text { Total } \\
\text { Number } \\
\text { of } \\
\text { Directives }\end{array}$} & \multicolumn{2}{|c|}{$\begin{array}{c}\text { Directives } \\
\text { Responded to } \\
\text { without } \\
\text { Instructor's } \\
\text { Prompts }\end{array}$} & \multicolumn{2}{|c|}{$\begin{array}{l}\text { Directives } \\
\text { Responded to } \\
\text { only with } \\
\text { Instructor's } \\
\text { Prompts }\end{array}$} & \multicolumn{2}{|c|}{$\begin{array}{c}\text { Directives to } \\
\text { which there } \\
\text { was No } \\
\text { Response }\end{array}$} \\
\hline & & & Total & $\%$ & Total & $\%$ & Total & $\%$ \\
\hline 1 & Nov 22015 & 105 & 64 & 60.95 & 37 & 35.23 & 4 & 3.80 \\
\hline 2 & Nov 52015 & 96 & 60 & 62.5 & 29 & 30.20 & 7 & 7.29 \\
\hline 3 & Nov 92015 & 138 & 95 & 68.84 & 32 & 23.18 & 11 & 7.97 \\
\hline 4 & Nov 122015 & 95 & 80 & 84.21 & 10 & 10.52 & 5 & 5.26 \\
\hline 5 & Nov 162015 & 118 & 93 & 78.81 & 20 & 16.94 & 5 & 4.23 \\
\hline
\end{tabular}




\begin{tabular}{|c|c|c|c|c|c|c|c|c|}
\hline 6 & Nov 19 2015 & 126 & 84 & 66.66 & 30 & 23.80 & 12 & 9.52 \\
\hline 7 & Nov 23 2015 & 152 & 119 & 78.28 & 26 & 17.10 & 7 & 4.60 \\
\hline 8 & Nov 30 2015 & 148 & 114 & 77.02 & 31 & 20.94 & 3 & 2.02 \\
\hline 9 & Dec 3 2015 & 104 & 78 & 75 & 25 & 24.03 & 1 & 0.96 \\
\hline 10 & Jan 4 2016 & 118 & 72 & 61.01 & 42 & 35.59 & 4 & 3.38 \\
\hline 11 & Jan 7 2016 & 72 & 56 & 77.77 & 14 & 19.44 & 3 & 4.16 \\
\hline 12 & Jan 11 2016 & 30 & 21 & 70 & 8 & 26.66 & 1 & 3.33 \\
\hline 13 & Jan 14 2016 & 73 & 56 & 76.71 & 15 & 20.54 & 2 & 2.73 \\
\hline 14 & Jan 25 2016 & 86 & 55 & 63.95 & 29 & 33.72 & 2 & 2.32 \\
\hline 15 & Jan 28 2016 & 88 & 70 & 79.54 & 17 & 19.31 & 1 & 1.13 \\
\hline 16 & Feb 11 2016 & 52 & 36 & 69.23 & 13 & 25 & 3 & 5.76 \\
\hline 17 & Mar 24 2016 & 98 & 76 & 77.55 & 20 & 20.40 & 2 & 2.04 \\
\hline 18 & Mar 31 2016 & 89 & 71 & 79.77 & 15 & 16.85 & 3 & 3.37 \\
\hline 19 & Apr 14 2016 & 70 & 57 & 81.42 & 10 & 14.28 & 3 & 4.28 \\
\hline
\end{tabular}

Figure 2 presents an analysis of the progress made by the subject. The analysis shows that over time the subject improved in responses without instructor prompting. In addition, both the number of responses with prompting and the number of no-responses decrease over time. Interestingly, these measures suggest an improvement of the subject over 19 sessions of social training with the presence of the humanoid robot.

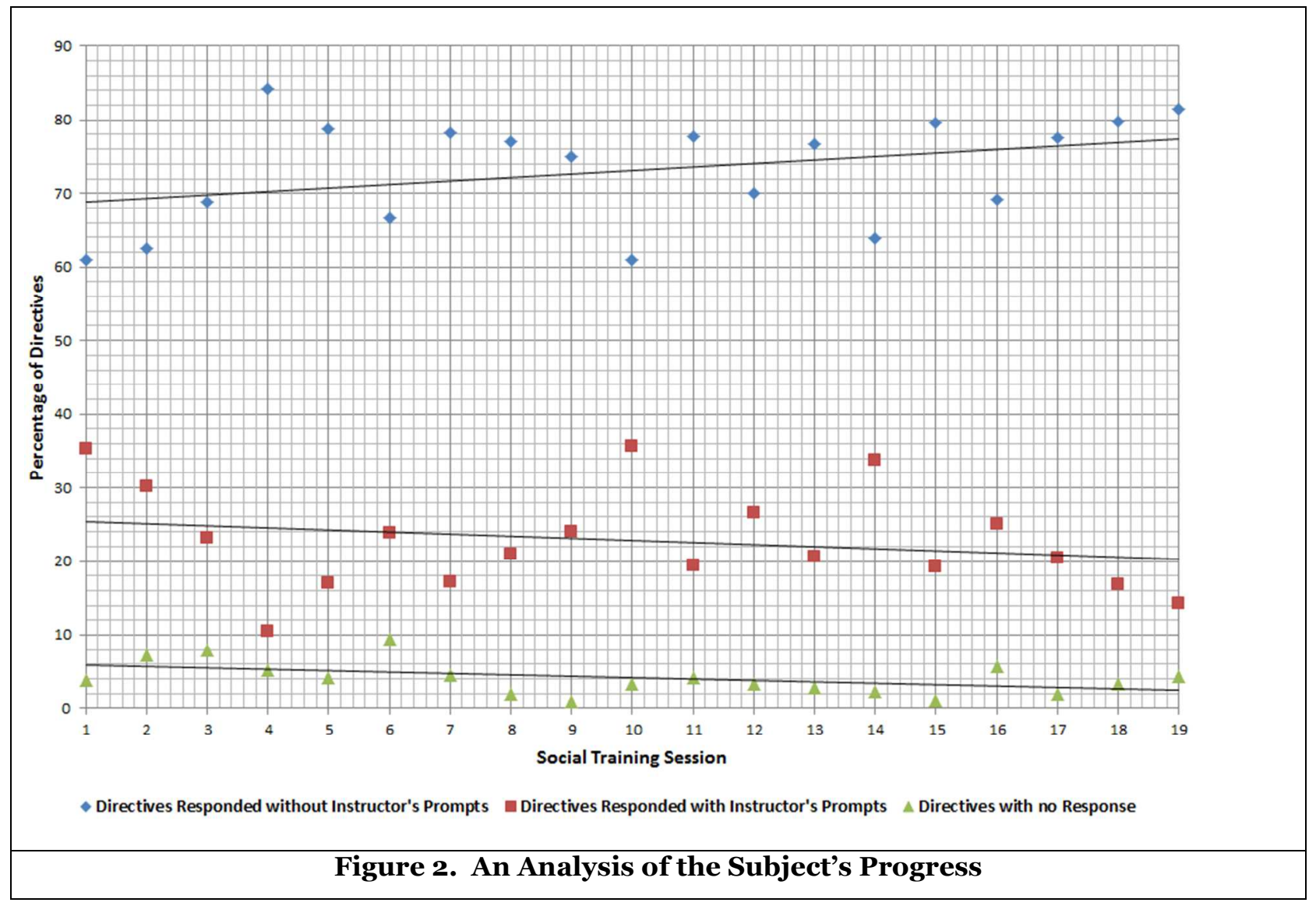


Despite the promises that the current study demonstrates for future research, the authors appreciate the lack of comparison of the results when the control variable of robots with no facial expression is not contributing. The authors acknowledge the limitations that it creates for the conclusions. However, the current study is a pilot targeting the demonstration of the relevance in the idea of lean robotic communication for autistic training sessions.

\section{Discussion, Future Work and Conclusion}

With the advances in robotic technologies, research in ASD intervention has shown enthusiasm towards using the technology in ASD therapy. While there is a body of literature that reports ASD children improving social skills by using robots in training sessions (Anzalone et al., 2014; Dautenhahn, 2007; Wainer et al., 2014), some studies cannot find any significant benefit (Yee et al., 2012; Yin and Tung, 2013; Zheng et al., 2013). Children with ASD suffer from difficulties in understanding the facial expressions occurring during communications. This results in inability of the children to maintain interest in the training sessions. Scassellati (2007) highlights the potential of robots to create standardized communications without complex structures of facial expressions in training sessions. A systematic review of the literature (Pennisi et al., 2015; ) demonstrates the potential of robots supporting training in social skills for ASD children. However, our research of the literature showed that more collaboration is needed among the robotics community and ASD community (Kim et al., 2012).

The current study attempted to address the above gap in the literature, and demonstrated, that in nineteen training sessions, an autistic child improved in responses to the directives given by a humanoid robot with no facial expression. This result provides positive support that using a humanoid robot with no facial expressions can improve social performance. This has strong practical and theoretical implications $\mathrm{n}$ children with ASD. While we acknowledge that this study has only one subject, and thus it is hard to generalize the current findings, the study demonstrates a proof of concept for a larger trial. Further, the concept of a robot with no facial expression has demonstrated a potential that warrants further investigation into how humanoid robots can be utilized by clinicians and ASD instructors to improve the social interactions in children with ASD.

Interestingly, the results in the present study also support the idea of lean communication, and are in contrast to the proposed constructs of media fitness as well as its main assumption. This study indicates that media fitness cannot be defined based only on characteristics of tasks and media, but also needs to take into consideration, the characteristics of the receiver (e.g. in the case of this work, the child with autistism) and her/his cognitive and social abilities. The present study has shown that a robot without facial expression can significantly improve communication and improve the performance of the receiver.

The authors are planning further research that incorporates the cognitive and social abilities of receivers in defining the media-fitness for different types of communication. The proposed research will add an extra construct to media richness theory and the concept of media fitness. Towards this end, the authors plan to adopt the clinically used Social Responsiveness Scale (SRS) (Constantino and Gruber, 2007). This scale can measure social deficits of individuals, where SRS scores reflect various levels of social functioning, e.g. social awareness, social cognition, social communication, social motivation, and restricted interests and repetitive behavior. These scales, along with task characteristics, will be used to assess the richness of the media, while performance and satisfaction measures will be used to indicate media fitness. The performance and satisfaction measures will be similar to those in this study, viz. the number of responses without prompting, the number only with prompting, and no-responses. Autism can be defined by a curve of 'richness of media' (starting with o) against 'number of interactions engaged'. The authors expect and at this stage are designing a pilot study, which demonstrates that the curve would be a negatively sloping asymptotic curve. A high level of autism is indicated by the curve starting from very low number of interactions and a very steep slope reaching o interactions at a very low level of richness. Improvement is indicated by both climbing up on the y-axis (number of interactions) and a less steep slope.

\section{Future Work}

Future research will involve the development of an instrument for measuring the degree to which the children with ASD can involve in social communications. The theory of lean communication can be used for measuring the ability of autistic children in social involvement. The point at which (i.e. number of 
interactions) a subject stops interacting with the robot is an indicator of the autism degree: the earlier a subject stops interacting, the higher the level of autism. Anzalone et al (2014) believe that current measures of the level of autism rely on subjective assessments of trained specialists. The tool developed here can be validated against existing subjects relying on subjective assessments and employed as an additional piece of information in assessing the level of autism of a subject, and even be employed by nontrained specialists to assess the level of autism. By the same token, the robotic tool can also be employed to measure changes in the level of autism over time, or as a consequence of intervention such as a social skill training program. The robotic tool can be programmed to increase the level of richness as an additional measure to assess the level of autism. This too can be employed to measure the level of autism as well as improvement in subjects. It can also be employed as a training tool by progressively increasing the richness of communication. The current pilot study presented in this paper, limited to the number of subjects, provides a proof of concept in which the autistic child showed some progress in the pilot study. Therefore, the potential for such training programs can be seen.

Although the sessions were recorded, the current paper does not report an in-depth analysis of the subject's reactions to the robot other than responses to the robot's directives. We plan to analyze the videos and report further qualitative observations of the subject's behavioral reactions to the robot. The present study does not provide a sufficient level of depth on socio-economic issues such as cost, technical support, and mainstream viability of the intervention method. In addition, the findings do not show how a subject's external activities affect the subject's performance during robot therapy and vice versa, e.g. home life, ordinary classroom performance, playground activities, and contemporaneous ASD interventions such as music therapy and occupational therapy. These limitations suggest a host of future socioeconomic research questions.

\section{References}

Anzalone, S.M., Tilmont, E., Boucenna, S., Xavier, J., Jouen, A.-L., Bodeau, N., Maharatna, K., Chetouani, M., Cohen, D., Group, M.S., 2014. How children with autism spectrum disorder behave and explore the 4-dimensional (spatial $3 \mathrm{~d}+$ time) environment during a joint attention induction task with a robot. Res. Autism Spectr. Disord. 8, 814-826.

Association of Autistic Patients., 2015. Diagnostic and Statistical Manual of Mental Disorders. DSM--5. ISBN 978--0--89042--554--1(en prensa).

Bellini, S., Peters, J.K., 2008. Social skills training for youth with autism spectrum disorders. Child Adolesc. Psychiatr. Clin. N. Am. 17, 857-873.

Bogdashina, O. (2016). Sensory perceptual issues in autism and asperger syndrome: Different sensory experiences-different perceptual worlds. Jessica Kingsley Publishers.

Bubaš, G., 2001. Computer mediated communication theories and phenomena: Factors that influence collaboration over the Internet, in: 3rd CARNet Users Conference. Citeseer.

Cabibihan, J.-J., Javed, H., Ang Jr, M., Aljunied, S.M., 2013. Why robots? A survey on the roles and benefits of social robots in the therapy of children with autism. Int. J. Soc. Robot. 5, 593-618.

Cappadocia, M.C., Weiss, J.A., Pepler, D., 2012. Bullying experiences among children and youth with autism spectrum disorders. J. Autism Dev. Disord. 42, 266-277.

Constantino, J.N., Gruber, C.P., 2007. Social responsiveness scale (SRS). Western Psychological Services Los Angeles, CA.

Daft, R.L., Lengel, R.H., 1986. Organizational information requirements, media richness and structural design. Manag. Sci. 32, 554-571.

Dautenhahn, K., 2007. Socially intelligent robots: dimensions of human-robot interaction. Philos. Trans. R. Soc. Lond. B Biol. Sci. 362, 679-704.

Diehl, J. J., Schmitt, L. M., Villano, M., \& Crowell, C. R. (2012). The clinical use of robots for individuals with autism spectrum disorders: A critical review. Research in autism spectrum disorders, 6(1), 249262.

Gilbert, Richard O. "6.5 Sen's Nonparametric Estimator of Slope." Statistical Methods for Environmental Pollution Monitoring, John Wiley and Sons (1987): 217-219.

Hobson, R.P., 1986. The autistic child's appraisal of expressions of emotion. J. Child Psychol. Psychiatry 27, 321-342. 
Kim, E.S., Paul, R., Shic, F., Scassellati, B., 2012. Bridging the research gap: Making HRI useful to individuals with autism. J. Hum.-Robot Interact. 1.

Kroeger, K.A., Schultz, J.R., Newsom, C., 2007. A comparison of two group-delivered social skills programs for young children with autism. J. Autism Dev. Disord. 37, 808-817.

Lewis, L., Charron, N., Clamp, C., Craig, M., 2016. Soft Systems Methodology As a Tool to Aid a Pilot Study in Robot-Assisted Therapy, in: The Eleventh ACM/IEEE International Conference on Human Robot Interation, HRI '16. IEEE Press, Piscataway, NJ, USA, pp. 467-468.

Otero, T.L., Schatz, R.B., Merrill, A.C., Bellini, S., 2015. Social skills training for youth with autism spectrum disorders: a follow-up. Child Adolesc. Psychiatr. Clin. N. Am. 24, 99-115.

Pennisi, P., Tonacci, A., Tartarisco, G., Billeci, L., Ruta, L., Gangemi, S., Pioggia, G., 2015. Autism and social robotics: A systematic review. Autism Res.

Puyon, M., Giannopulu, I., 2013. Emergent emotional and verbal strategies in autism are based on multimodal interactions with toy robots in free spontaneous game play, in: RO-MAN, 2013 IEEE. IEEE, pp. 593-597.

Ricks, D. J., \& Colton, M. B. (2010). Trends and considerations in robot-assisted autism therapy. Proceedings of IEEE international conference on robotics and automation.Robins, B., Dautenhahn, K., Te Boekhorst, R., \& Billard, A. (2005). Robotic assistants in therapy and education of children with autism: can a small humanoid robot help encourage social interaction skills?. Universal Access in the Information Society, 4(2), 105-120.

Robins, B., Dautenhahn, K., \& Dubowski, J. (2006). Does appearance matter in the interaction of children with autism with a humanoid robot?. Interaction Studies, 7(3), 509-542.

Robins, B., Dautenhahn, K., \& Dickerson, P. (2009, February). From isolation to communication: a case study evaluation of robot assisted play for children with autism with a minimally expressive humanoid robot. In Advances in Computer-Human Interactions, 2009. ACHI'09. Second International Conferences on (pp. 205-211). IEEE.

Russo-Ponsaran, N.M., Evans-Smith, B., Johnson, J., Russo, J., McKown, C., 2015. Efficacy of a Facial Emotion Training Program for Children and Adolescents with Autism Spectrum Disorders. J. Nonverbal Behav. 126.

Scassellati, B., 2007. How social robots will help us to diagnose, treat, and understand autism, in: Robotics Research. Springer, pp. 552-563.

Scassellati, B., Admoni, H., \& Matarić, M. (2012). Robots for use in autism research. Annual review of biomedical engineering, 14, 275-294.

Suh, K.S., 1999. Impact of communication medium on task performance and satisfaction: an examination of media-richness theory. Inf. Manage. 35, 295-312.

Wainer, J., Robins, B., Amirabdollahian, F., Dautenhahn, K., 2014. Using the humanoid robot KASPAR to autonomously play triadic games and facilitate collaborative play among children with autism. Auton. Ment. Dev. IEEE Trans. On 6, 183-199.

Yee, A.W.H., Kee, T.Y., Limbu, D.K., Jian, A.T.H., Dung, T.A., Yuen, A.W.C., 2012. Developing a robotic platform to play with pre-school autistic children in a classroom environment, in: Proceedings of the Workshop at SIGGRAPH Asia. ACM, pp. 81-86.

Yin, T.-C., Tung, F.-W., 2013. Design and evaluation of applying robots to assisting and inducing children with autism in social interaction, in: Universal Access in Human-Computer Interaction. User and Context Diversity. Springer, pp. 524-533.

Zheng, Z., Zhang, L., Bekele, E., Swanson, A., Crittendon, J.A., Warren, Z., Sarkar, N., 2013. Impact of robot-mediated interaction system on joint attention skills for children with autism, in: Rehabilitation Robotics (ICORR), 2013 IEEE International Conference on. IEEE, pp. 1-8. 